\title{
Denucleation rates during Ostwald ripening: Distribution kinetics of unstable clusters
}

\author{
Giridhar Madras* \\ Department of Chemical Engineering, Indian Institute of Science, Bangalore 560 012, India \\ Benjamin J. McCoy \\ Department of Chemical Engineering and Materials Science, University of California, Davis, \\ California 95616
}

\begin{abstract}
During Ostwald ripening in vapor-liquid or liquid-solid systems, unstable clusters, which are smaller than the critical nucleus size, rapidly disappear. This denucleation process is distinct from the reversible dissolution of stable clusters that are larger than the critical size but are more soluble than larger clusters because of the Gibbs-Thomson effect. Thus, ripening involves larger clusters growing, smaller clusters shrinking, and unstable clusters rapidly disintegrating. We show how the denucleation rate can be estimated based on the appropriate cluster distribution dynamics (population balance) equations. Monomer addition and dissociation from an unstable cluster occur at rates determined by the energy profile, which is formulated with classical nucleation concepts. Effectively, the cluster loses free energy as monomers dissociate, and falls down the energy stair steps. Numerical results for the distribution dynamics equations reveal the influence of the minimum number of dimensionless parameters, and demonstrate that denucleation is very fast compared to the ripening rate.
\end{abstract}

\section{INTRODUCTION}

The final stage of a condensation phase transition is Ostwald ripening, or coarsening, whereby smaller, unstable clusters dissolve, giving up their mass so that larger stable clusters can grow. For liquid-solid condensation the clusters are particles that precipitate, for example, crystals; for vapor-liquid condensation the clusters are droplets. As the supersaturation approaches unity, the size of the unstable clusters increases. The general understanding is that eventually all but one stable cluster will have vanished. ${ }^{1,2}$ The vanishing rate of these unstable clusters has not been systematically examined, and in many theories of ripening, has been ignored.

Cluster dissolution and growth in ripening is closely related to similar processes in homogeneous nucleation. Unstable clusters can exist only as transient fluctuations caused by monomer addition and dissociation. The Gibbs-Thomson equation stipulates that the solubility (or dissociation tendency) is greater for smaller clusters owing to their larger surface free energy per volume or mass. According to the classical picture, ${ }^{3}$ nucleation occurs by one-at-a-time deposition of monomers that increase the free energy of a cluster until the free energy barrier at the critical cluster (nucleus) size is surmounted. Incorporating the sum of surface and volume formation free energy contributions, the free energy barrier increases and shifts to larger clusters as the supersaturation decreases due to monomer deposition. Once past the maximum free energy, the stable cluster decreases its energy

\footnotetext{
*Author to whom correspondence should be addressed. Phone: 91-080-309-2321; Fax: 91-080-360-0683;

electronic mail: giridhar@chemeng.iisc.ernet.in
}

by adding more monomers, growing according to a driving force that decreases as the supersaturation declines to its equilibrium value. Differentiating between stable and unstable clusters, this view has important consequences for Ostwald ripening and for phase transition dynamics in general.

Denucleation can occur during growth and ripening processes when the supersaturation decreases, causing the critical nucleus size to increase. Any cluster smaller than critical size is unstable and will tend to dissolve spontaneously. Although the problem of quantifying the homogeneous nucleation rate has been studied extensively, almost no attention has been directed toward the denucleation problem associated with Ostwald ripening. In fact, most theories of ripening neglect denucleation and allow clusters down to zero size to be present in the cluster size distribution. ${ }^{2,4-6}$ Madras and McCoy ${ }^{7}$ included denucleation in their ripening models, assuming that the rate of dissolution of unstable clusters is instantaneous. Such an infinite rate is tantamount to assuming the slowest rate (growth or dissociation in the stable region) completely dominates the ripening process. ${ }^{2}$ Although this assumption seems reasonable, especially considering the success of ripening models based on it, confirmation of the assumption remains a gap in the theory. Our aim here is to estimate the denucleation rate for clusters of size less than the stable cluster. Such an estimate must account for the interaction of thermodynamics, kinetics, and dynamics during phase transitions.

We follow tradition in using thermodynamic properties such as interfacial energy, formation free energy, and mass density for very small clusters assumed to be spherical, recognizing the problem in applying such concepts for clusters consisting of fewer than ten monomers. ${ }^{8}$ If we consider that 


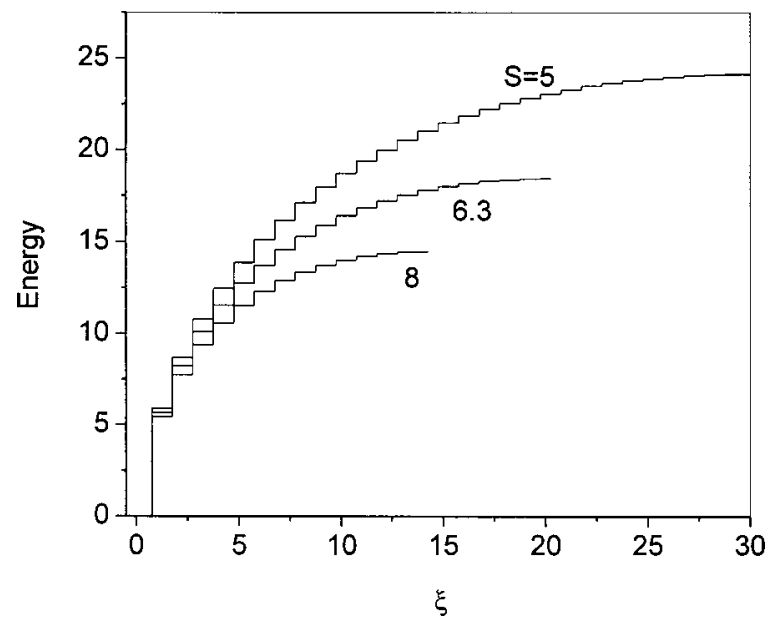

FIG. 1. Energy diagram, Eq. (2.6), for $\omega=5$ and $S=6.3$ and 2 showing the unstable-cluster stair steps. The energy maximum appears when the number of monomers in the cluster is $\xi^{*}=(\omega / \ln S)^{3}$, Eq. (2.8).

the critical cluster comprises many monomers, however, then the effect of the approximate treatment for small clusters becomes less significant. Estimates of denucleation rates based on this approximation will certainly be considerably improved over the assumption of instantaneous rates. ${ }^{7}$

Metastable solutions tend to condense when the supersaturated monomers aggregate one-by-one to form dimers, trimers, tetramers, and so on. The attractive forces that hold these clusters together are opposed by thermal agitation. Decreasing the temperature increases the cohesive effect of attractive energy relative to thermal energy. The competition between these effects is expressed by the total cluster energy, which is the sum of interfacial and volume terms. ${ }^{9}$ Homogeneous nucleation can occur when this cluster free energy reaches the maximum, and then volume-dominated energy can decrease by further monomer addition (stable growth). By adding monomers one-at-a-time, the cluster free energy rises by a discrete stair-climbing process until it reaches the maximum, critical energy (see Fig. 1). Clusters smaller than the critical size are dominated by interfacial energy and are unstable, existing only as energy fluctuations. The reversible growth and dissolution of unstable clusters gives rise to a cluster size distribution in a manner similar to chain polymerization. This picture of reversible monomer addition to or dissociation from unstable clusters enables us to quantitatively represent how a size distribution of clusters will evolve in time. We will develop the equations that govern the cluster size distribution under unstable conditions, thus representing how rapidly any such distribution will denucleate as energy declines until clusters are dissolved to monomer.

Some theories ${ }^{10,11}$ of homogeneous nucleation have been based on master equations for the stair-climbing process pictured in Fig. 1. Master equations for population transitions are inherently first-order in cluster distribution, ${ }^{12}$ such that the bimolecular interaction of monomer and cluster is approximated by pseudo-first-order kinetics. Gerlach ${ }^{13}$ formulated a population dynamics equation with growth and dissociation rate coefficients varying with cluster size. Such chemical kinetic views have inspired approaches to homoge- neous nucleation, ${ }^{14}$ as well as the present model for treating denucleation.

Our approach has the objective of evaluating rates of transitions or fluctuations for monomer dissociation from an unstable cluster, defined as a cluster of size less than the critical nucleus size. We hypothesize that such dynamics can be represented quantitatively by a discrete population balance, or distribution dynamics equation, which is a generalization of the master equation. The mathematical model is for a vessel in which clusters of unstable size are disintegrating. The cluster size distribution obeys a continuous distribution dynamics equation that can be transformed to discrete form for finite numbers of clustered monomers. ${ }^{15}$ Energy expressions enter into the temperature dependence of the rate coefficients for monomer addition and dissociation, which are related by microscopic reversibility for stable clusters but not for the unstable clusters considered here. To develop rate expressions for dissolution, the Gibbs-Thomson (Kelvin) equation relates surface and thermal energy to solubility and thus supersaturation. The equations for cluster dissolution take on simple forms when cast in dimensionless variables, and are readily solved numerically. For constant-temperature denucleation, the size distribution of unstable clusters depends on but five parameters: the supersaturation $S$, the ratio of surface to thermal energy $\omega$, two exponents $\lambda$ and $\nu$ of cluster mass for dissociation and deposition rates, and the ratio of dissociation to deposition rate $\beta$. Of these, $S$ and $\omega$ are the key parameters that determine the order of magnitude of the denucleation rate.

\section{CLUSTER ENERGY AND DENUCLEATION RATE}

We base our approach on the Gibbs capillarity approximation, ${ }^{16}$ which utilizes the sum of surface energy and formation free energy for a spherical cluster of radius $r_{c}$

$$
W(x)=4 \pi r_{c}^{2} \sigma-(4 / 3) \pi r_{c}^{3}\left(\rho_{c} / x_{m}\right) k_{B} T \ln S .
$$

Here, $\sigma$ is the cluster interfacial energy, $\rho_{c}$ is the cluster mass density, $x_{m}$ is the monomer mass, and $-k_{B} T \ln S$ is the cluster energy difference between the two phases in terms of supersaturation,

$$
S=m^{(0)} / m_{\infty}^{(0)}
$$

where $m^{(0)}$ is the monomer molar concentration and the subscript $\infty$ refers to its equilibrium value for a flat surface. The applicability of continuum thermodynamic properties such as interfacial energy $\sigma$ and cluster density $\rho_{c}$ is obviously questionable for very small clusters. Indeed, even if these properties can be defined, one expects $\sigma$ and $\rho_{c}$ would depend on the number of monomers in such clusters. In the current computations, we consider many steps on the stairs, so that the activated fluctuations at the lower few steps will not dominate the overall cluster size distribution evolution, and hence we will take $\sigma$ and $\rho_{c}$ as constants of $x$. This will serve to evaluate the proposed denucleation model, and to illustrate its implementation. Then the cluster mass $x$ is related to the condensed-phase mass density $\rho_{c}$ and cluster radius $r_{c}$ by 
$x=(4 / 3) \pi r_{c}^{3} \rho_{c}$. An alternative approach is to express $W(x)$ as an increasing function of cluster size with two undetermined parameters. ${ }^{14}$

For the assumed spherical cluster the local-equilibrium interfacial concentration at the cluster surface is given by the Gibbs-Thomson equation, ${ }^{2}$

$$
m_{\mathrm{eq}}^{(0)}=m_{\infty}^{(0)} \exp (\Omega),
$$

where

$$
\Omega=2 \sigma x_{m} / r_{c} \rho_{c} k_{B} T,
$$

in terms of monomer volume $x_{m} / \rho_{c}$, the Boltzmann constant $k_{B}$, and absolute temperature $T$. Thus smaller clusters are more soluble than larger clusters. For vapor-liquid processes, $m_{\infty}^{(0)}=P_{\infty}(T) / R T$ is expressed in terms of the standard vapor pressure for a flat surface, $P_{\infty}(T)$. Equation (2.4) has been verified experimentally ${ }^{3}$ for water clusters as small as $10^{-5} \mathrm{~cm}$ and may be valid ${ }^{17}$ at $<10^{-6} \mathrm{~cm}$. Frenkel ${ }^{8}$ argued that the thermodynamic surface-energy expression was satisfactory down to clusters of ten molecules. Although corrections for small clusters can be written, ${ }^{16}$ the classical approximation serves to illustrate the current approach.

The mathematical manipulations are streamlined if we define dimensionless variables,

$$
\xi=x / x_{m} \text { and } \omega=\left[(4 \pi / 3)\left(x_{m} / \rho_{c}\right)^{2}\right]^{1 / 3} 2 \sigma / k_{B} T,
$$

where $\omega$ represents the ratio of surface energy to thermal energy. Equations (2.1) and (2.4) are recast as

$$
\Gamma(\xi) \equiv W(x) / k_{B} T=(3 / 2) \omega \xi^{2 / 3}-\xi \ln S
$$

and

$$
\Omega(x)=\omega / \xi^{1 / 3} .
$$

Note that $\xi$ is the number of monomers in a cluster. To show the decreasing stair-step heights as $\xi$ increases, Eq. (2.6) is plotted in Fig. 1 for $\omega=5$ and various values of $S$. For the smaller supersaturation, the cluster size at the energy maximum is larger. Classical homogeneous nucleation theory, and thus our denucleation model, is based on energy fluctuations, which for nucleation allow cluster energy to reach the maximum (activation) energy. We see that $W(x)$ is zero at $\xi=0$ and has its maximum at

$$
\xi^{*}=(\omega / \ln S)^{3} .
$$

Thus by Eq. (2.6) the maximum energy is

$$
\Gamma^{*}=(1 / 2) \omega^{3} /(\ln S)^{2} .
$$

The dimensionless quantities, Eqs. (2.5)-(2.9), are similar to expressions defined previously. ${ }^{18}$

\section{CLUSTER DISTRIBUTION KINETICS}

For denucleation, the unstable cluster size distribution is defined by $c(x, t) d x$, representing the concentration of clusters at time $t$ in the differential mass range $(x, x+d x)$. Moments are defined as integrals over the mass, where the limits of integration are the dimer mass, $2 x_{m}$, and critical nucleus mass, $x^{*}$,

$$
c^{(n)}(t)=\int c(x, t) x^{n} d x
$$

The zeroth moment, $c^{(0)}(t)$, and the first moment, $c^{(1)}(t)$, of clusters are the time-dependent molar (or number) concentration and the mass concentration (mass/volume), respectively. The ratio of the two is the average cluster mass, $c^{\text {avg }}=c^{(1)} / c^{(0)}$. The variance, $c^{\mathrm{var}}=c^{(2)} / c^{(0)}-\left[c^{\mathrm{avg}}\right]^{2}$, and the polydispersity index, $c^{\mathrm{pd}}=c^{(2)} c^{(0)} / c^{(1) 2}$, are measures of the cluster polydispersity. The molar concentration, $m^{(0)}(t)$, of solute monomer of molecular weight $x_{m}$ is the zeroth moment of the monomer distribution $m(x, t)=m^{(0)}(t) \delta\left(x-x_{m}\right)$.

The process by which monomers of mass $x^{\prime}=x_{m}$ are reversibly added to or dissociated from a cluster of mass $x$ is similar to a chain polymerization, or depolymerization, reaction, $^{7}$

$$
\mathbf{C}(x)+\mathbf{M}\left(x^{\prime}\right) \underset{k_{d}(x)}{\stackrel{k_{g}(x)}{\rightleftarrows}} \mathbf{C}\left(x+x^{\prime}\right),
$$

where $\mathbf{C}(x)$ is the cluster of mass $x$ and $\mathbf{M}\left(x^{\prime}=x_{m}\right)$ is the monomer. This process intrinsically conserves mass, and is naturally expressed in terms of mass $x$ rather than cluster radius $r$. When many monomers make up a cluster, the lower limit of integration, $2 x_{m}$, can be replaced by 0 . Similar to polymerization kinetics, the balance equations governing the cluster distribution, $c(x, t)$, and the monomer distribution, $m(x, t)$, are based on mass conservation.

The population balance equations, for a well-mixed vessel of volume $V$ with flow rate $Q$ having average residence time $\tau_{r}=V / Q$, and with the inlet distributions for crystals and solute $c_{i n}(x)$ and $m_{i n}(x)$, respectively, are given by

$$
\begin{aligned}
\partial c(x, t) / \partial t= & -k_{g}(x) c(x, t) \int_{0}^{x^{*}} m\left(x^{\prime}, t\right) d x^{\prime} \\
& +\int_{0}^{x^{*}} k_{g}\left(x-x^{\prime}\right) c\left(x-x^{\prime}, t\right) m\left(x^{\prime}, t\right) d x^{\prime} \\
& -k_{d}(x) c(x, t)+\int_{x}^{x^{*}} k_{d}\left(x^{\prime}\right) c\left(x^{\prime}, t\right) \\
& \times \delta\left(x-\left(x^{\prime}-x_{m}\right)\right) d x^{\prime}+\left(c_{i n}-c\right) / \tau_{r}
\end{aligned}
$$

and

$$
\begin{aligned}
\partial m(x, t) / \partial t= & -m(x, t) \int_{0}^{x^{*}} k_{g}\left(x^{\prime}\right) c\left(x^{\prime}, t\right) d x^{\prime} \\
& +\int_{x}^{x^{*}} k_{d}\left(x^{\prime}\right) c\left(x^{\prime}, t\right) \delta\left(x-x_{m}\right) d x^{\prime} \\
& +\left(m_{i n}-m\right) / \tau_{r} .
\end{aligned}
$$

For a batch vessel with no flow, $1 / \tau_{r}=0$. According to the molecularity (mass action) of Eq. (3.2), additional reactions are second-order in $c(x, t)$ and $m(x, t)$, whereas dissociation reactions are first-order in $c(x, t)$. Initial conditions for Eqs. (3.3) and (3.4) are $c(x, t=0)=c_{0}(x)$ and $m(x, t=0)$ $=m_{0}^{(0)} \delta\left(x-x_{m}\right)$. 
The cluster size distribution evolves according to Eq. (3.3), which becomes, when the integrations over the Dirac distributions are performed, the finite-difference differential equation,

$$
\begin{aligned}
\partial c(x, t) / \partial t= & -k_{g}(x) c(x, t) m^{(0)}+k_{g}\left(x-x_{m}\right) \\
& \times c\left(x-x_{m}, t\right) m^{(0)}-k_{d}(x) c(x, t) \\
& +k_{d}\left(x+x_{m}\right) c\left(x+x_{m}, t\right) .
\end{aligned}
$$

Equation (3.5) shows that $c(x, t)$ increases by addition of mass $x_{m}$ to the reactant of mass $\left(x-x_{m}\right)$ and decreases by the loss of reactant of mass $x$. The dissociation of mass $x_{m}$ from reactant of mass $\left(x+x_{m}\right)$ increases $c(x, t)$ while the loss of reactant of mass $x$ decreases $c(x, t)$. It is not necessary to assume arbitrarily that forward rate coefficients are first-order in monomer concentration, ${ }^{19}$ since the dependence on $m^{(0)}$ appears as a natural consequence of Eq. (3.4). Equation (3.5) can be expanded for $x_{m} \ll x$ to convert the differences into differentials, ${ }^{7}$ and to obtain a partial differential (Fokker-Planck) equation for growth and dissolution.

In liquid-solid phase transitions, a monomer that attaches to a cluster diffuses through the solution to the cluster surface by surmounting the activation energy, $W(x)$. Such diffusion-controlled reactionlike processes ${ }^{20,21}$ can be represented by

$$
k_{g}(x)=\gamma x^{\lambda} e^{-W(x) / R T},
$$

where $\lambda=1 / 3$. The $1 / 3$ power on $x$ represents diffusioncontrolled ripening, ${ }^{22}$ and the exponential $x$ dependence represents the activated step at each energy level for each monomer added.

When growth is limited by monomer attachment and dissociation at the cluster surface, ${ }^{22,23}$ the rate coefficient may be proportional to the cluster surface area, $k_{g} \propto r^{2}$, so that we can write [in Eq. (3.6)] $\lambda=2 / 3$ for surface-controlled growth. If the deposition is independent of the surface area, then $\lambda$ $=0$. Other values of $\lambda$ may be realistic for complex and combined rate processes.

Expressions for dissociation are considered not to have an activation barrier (or possibly one very small compared with the energy steps that must be surmounted for attachment to occur). We allow, however, for the possibility that dissociation may depend on cluster size and thus write

$$
k_{d}(x)=\kappa x^{\nu} .
$$

The coefficients $\gamma$ and $\kappa$ in Eqs. (3.6) and (3.7) may depend on parameters such as temperature, and thus may be assumed to be constants in this work. Gerlach ${ }^{13}$ proposed that $k_{d}$ decreases monotonically with $x$, such that, for example, $\nu$ $=-1$ in Eq. (3.7). Unlike clusters that can evolve to equilibrium as supersaturation decreases to unity, the unstable clusters are not required to obey microscopic reversibility (detailed balancing). For clusters larger than the critical nuclei, detailed balancing is necessary, but such stable clusters do not enter into the present analysis. Corresponding to the energy landscape schematically illustrated in Fig. 1, for the unstable clusters $\left(\xi<\xi^{*}\right)$ the reaction progress is down the stair steps. Denucleation will be rapid as there are no free energy barriers to monomer dissociation from the unstable cluster. Furthermore, fluctuations that allow the unstable cluster to grow are impeded by the energy steps, $W(x)$.

Substituting expressions for $k_{g}(x)$ and $k_{d}(x)$, Eqs. (3.6) and (3.7), into Eq. (3.5) yields

$$
\begin{aligned}
\partial c(x, t) / \partial t= & \gamma m^{(0)}(t)\left[-x^{\lambda} e^{-W(x) / R T} c(x, t)\right. \\
& \left.+\left(x-x_{m}\right)^{\lambda} e^{-W\left(x-x_{m}\right) / R T} c\left(x-x_{m}, t\right)\right] \\
& +\kappa\left[-x^{\nu} c(x, t)+\left(x+x_{m}\right)^{\nu} c\left(x+x_{m}, t\right)\right] .
\end{aligned}
$$

The powers on $x$ are unequal for the growth and dissociation terms owing to lack of detailed balancing for these unstable clusters. The governing equation for the vapor concentration is

$$
\begin{aligned}
d m^{(0)} / d t= & -\gamma m^{(0)}(t) \int_{2 x_{m}}^{x^{*}} x^{\lambda} e^{-W(x) / R T} c(x, t) d x \\
& +\kappa \int_{2 x_{m}}^{x^{*}} x^{\nu} c(x, t) d x .
\end{aligned}
$$

Next we introduce the dimensionless time, cluster distribution, moments, and dissociation coefficient,

$$
\begin{aligned}
& \theta=t \gamma m_{\infty}^{(0)} x_{m}^{\lambda}, \quad C=c x_{m} / m_{\infty}^{(0)}, \quad C^{(n)}=c^{(n)} / m_{\infty}^{(0)} x_{m}^{n}, \\
& \beta=\kappa /\left(\gamma m_{\infty}^{(0)} x_{m}^{\lambda-\nu}\right) .
\end{aligned}
$$

Thus $\beta$ represents the ratio of dissociation to association rate parameters. From Eq. (3.1) the scaled moments are

$$
C^{(n)}(\theta)=\int_{2}^{\xi^{*}} C(\xi, \theta) \xi^{n} d \xi .
$$

Substitution of the scaled quantities into Eqs. (3.8) and (3.9) yields the fully dimensionless difference-differential equation,

$$
\begin{aligned}
\partial C(\xi, \theta) / \partial \theta= & S(\theta)\left[-\xi^{\lambda} e^{-\Gamma(\xi)} C(\xi, \theta)\right. \\
& \left.+(\xi-1)^{\lambda} e^{-\Gamma(\xi-1)} C(\xi-1, \theta)\right] \\
& +\beta\left[-\xi^{\nu} C(\xi, \theta)+(\xi+1)^{\nu} C(\xi+1, \theta)\right]
\end{aligned}
$$

and the integral-differential equation

$$
d S(\theta) / d \theta=\int_{2}^{\xi^{*}}\left[-\xi^{\lambda} e^{-\Gamma(\xi)}+\beta \xi^{\nu}\right] C(\xi, \theta) d \xi
$$

with initial conditions,

$$
S(\theta=0)=S_{0} \text { and } C(\xi, \theta=0)=C_{0}(\xi) .
$$

Equation (3.13) is to be solved simultaneously with the difference-differential Eq. (3.12), which has a tridiagonal form (only terms with $\xi-1, \xi$, and $\xi+1$ appear in each equation). Numerical computations are solutions of Eqs. (3.12)-(3.14) for clusters smaller than the critical size, $\xi$ $\leqslant \xi^{*}$. We recognize that a cluster with but one molecule is a monomer, so that $C(1, \theta)=S$. The cluster initial condition, $C_{0}(\xi)$, is taken to be a delta distribution in our computations for denucleation, and is therefore given by $C(\xi, \theta=0)$ $=\delta\left(\xi=C_{0}^{\mathrm{avg}}\right)$. 


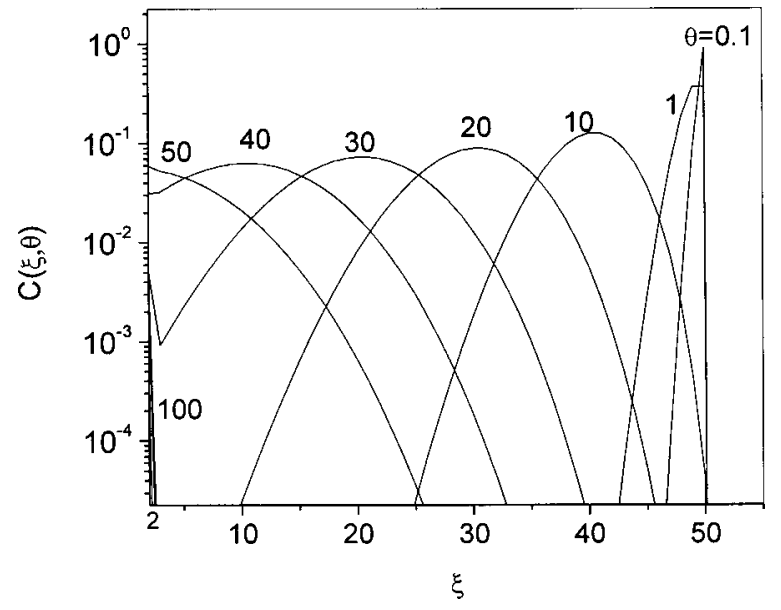

FIG. 2. Time evolution of the unstable CSD from an initial delta distribution at $C_{0}^{\mathrm{avg}}=50$. The parameters are $\beta=1, S=2, \omega=5$, and $\lambda=\nu=0$.

The computations are significantly simplified if the supersaturation is maintained constant with time. Such a steady supersaturation can be realized experimentally if monomer is continuously removed to make up for monomer that is released during denucleation. This will set a lower bound on the denucleation rate, as $S$ would otherwise decrease and thus increase the rate. The mass balance, Eq. (3.13), on monomer and cluster mass determines how much monomer is to be removed. Then the difference-differential Eq. (3.12), with $\Gamma(\xi)$ given by Eq. (2.6), can be readily solved for $C(\xi, \theta)$. The Runge-Kutta method was used to evaluate $C(\xi, \theta)$ at each time step sequentially for Eq. (3.12). A time step of 0.01 was chosen to ensure stability and accuracy of the numerical scheme. The cluster size distribution (CSD) is zero when $\xi \geqslant \xi^{*}$, and the cluster number concentration is calculated by integration of the nonzero CSD from 2 to $\xi^{*}$.

\section{COMPUTATIONAL RESULTS}

For illustrative computations we chose the following constant values: $C^{(0)}(\theta=0)=1, C^{\operatorname{avg}}(\theta=0)=2,10,50,100$, or $250 ; S=1.5,2$, or $3 ; \omega=4,5$, or $6 ; \beta=0.1,1$, or $10 ; \lambda$ $=0,1$, or 2 ; and $\nu=-1,0$, or 1 . The evolution of a distri-

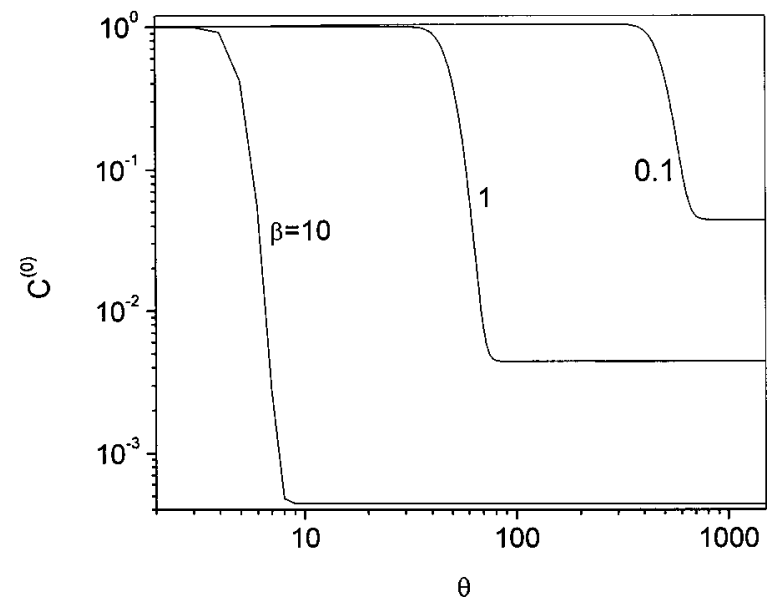

FIG. 3. Effect of $\beta$ on the variation of the dimensionless number of clusters, $C^{(0)}$, with time. The parameters are $S=2, C_{0}^{\mathrm{avg}}=50, \omega=5$, and $\lambda=\nu=0$.

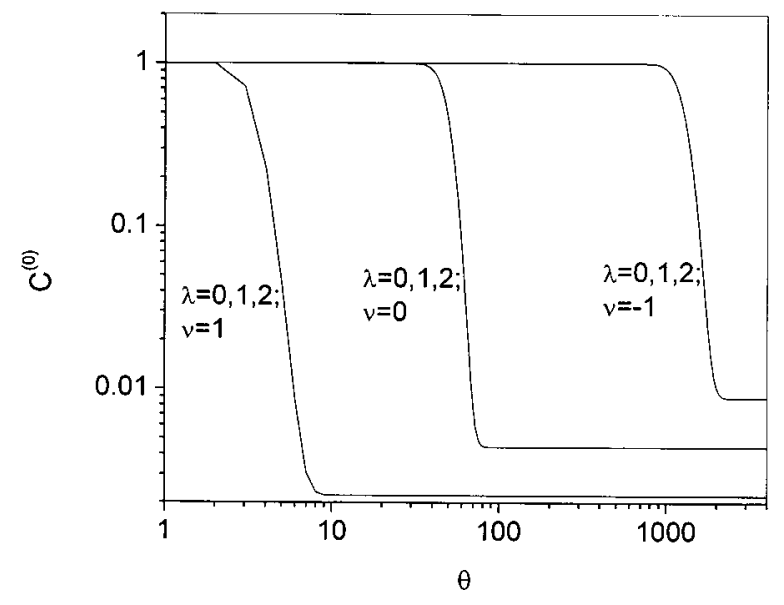

FIG. 4. Effect of rate exponents, $\lambda$ and $\mu$, on the variation of the number of clusters, $C^{(0)}$, with time. The parameters are $\beta=1, S=2, C_{0}^{\text {avg }}=50$, and $\omega$ $=5$.

bution is shown in Fig. 2 from an initial delta distribution at $C_{0}^{\text {avg }}=50$ to a final state where the concentration of the dimer is predominant with nearly complete dissolution of clusters other than the dimer. We are primarily concerned about how the number (or moles) of clusters, $C^{(0)}$, decreases with time. With an initial delta distribution, we choose the dimensionless zeroth moment, $C_{0}^{(0)}$, as unity and representative values for supersaturation, $S$, and the ratio of surface to thermal effects, $\omega$. The zeroth moment of the distribution (cluster moles) versus time is displayed in Fig. 3 for several values of $\beta$, which is the ratio of dissociation to association rate parameters, Eq. (3.10). Increasing $\beta$ proportionately decreases the time for the final distribution to be reached. Thus, the time-dependence of the cluster evolution is quite sensitive to the coefficient $\beta$ over most of the unstable region, $1<\xi<\xi^{*}$. This is in accord with the proposition that cluster dissociation dominates over cluster growth during denucleation. $^{7}$

The power $\nu$ on $x$ (or $\xi$ ) for the dissociation rate coefficient also overwhelms $\lambda$, the power for the growth coefficient, as shown in Fig. 4. Changes in $\lambda$ are negligible com-

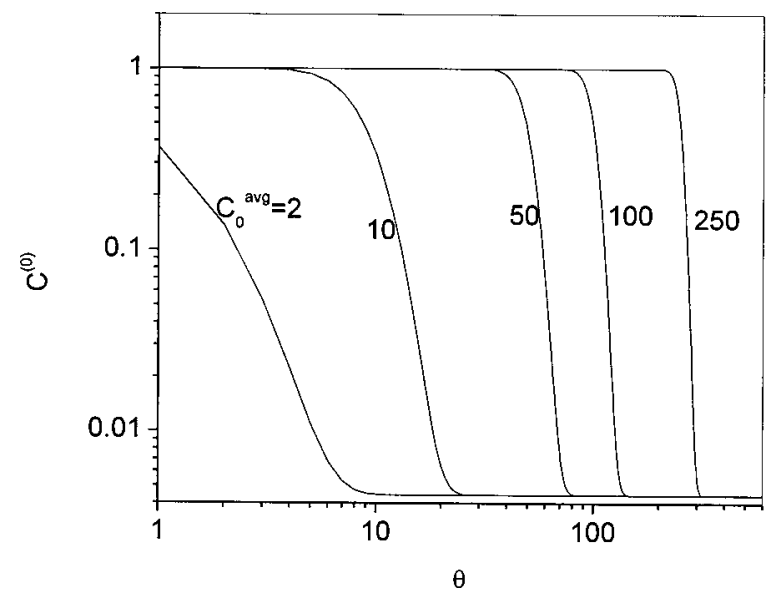

FIG. 5. Effect of the dimensionless initial average cluster size, $C_{0}^{\text {avg }}$, on the time variation of the number of clusters. The parameters are $\beta=1, S=2$, $\omega=5$, and $\lambda=\nu=0$. 


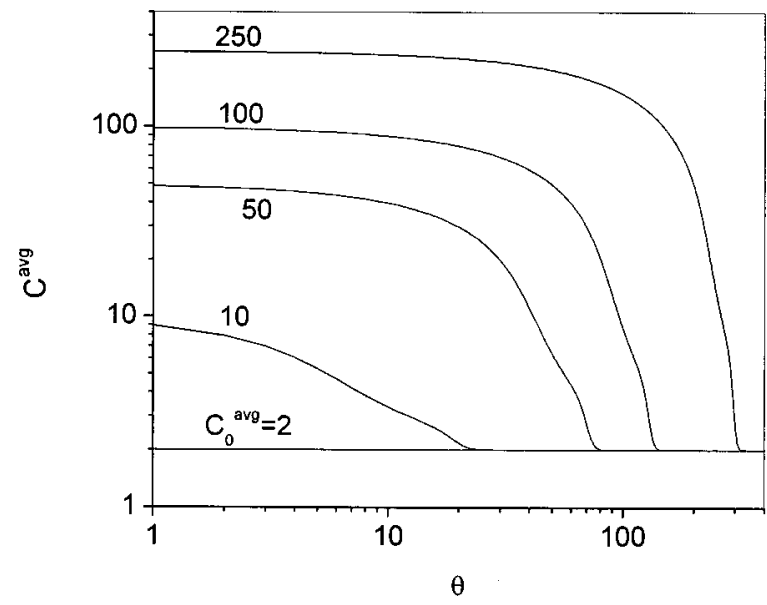

FIG. 6. Effect of the dimensionless initial average cluster size, $C_{0}^{\text {avg }}$, on the time variation of the average cluster size, $C^{\text {avg }}$. The parameters are $\beta=1$, $S=2, \omega=5$, and $\lambda=\nu=0$.

pared to the substantial effect of $\nu$, whereas increasing $\nu$ strongly increases the denucleation rate (Fig. 4). The influence of the initial average cluster size (Fig. 5) is significant. Small clusters dissolve quickly and larger clusters have an induction time. The larger clusters have a longer induction time because the final average cluster size is nearly two (the concentration of the dimer, although small, is much higher than the concentration of other clusters). This is apparent from the time evolution of the average cluster size (Fig. 6). The final CSD is predominately dimer with the concentration of other clusters being nearly equal to zero. The nature of the final CSD can be discerned mathematically by equating Eq. (3.12) to zero for $2 \leqslant \xi \leqslant \xi^{*}$. The final state of the dimer distribution, $C(2)$, obtained by equating Eq. (3.12) to zero for $\xi=2$, is given by $\left(\beta C(3)+S^{2} e^{-\Gamma(1)}\right) /\left(\beta+S e^{-\Gamma(2)}\right)$. Because $e^{-\Gamma(\xi)}$ decreases with increasing $\xi$, the the dimer concentration is much larger than $C(\xi>2)$. Thus the dimer concentration greatly influences the total number concentration.

The supersaturation, here assumed to be constant, affects only the final number of clusters (Fig. 7), and not the rate of denucleation. This is in keeping with the realization that dis-

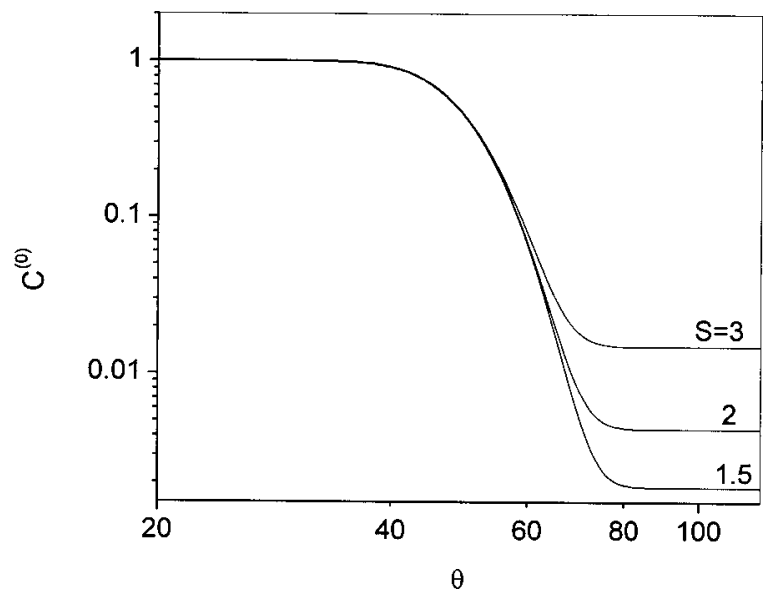

FIG. 7. Effect of the supersaturation, $S$, on the time variation of the number of clusters. The parameters are $\beta=1, C_{0}^{\text {avg }}=50, \omega=5$, and $\lambda=\nu=0$.

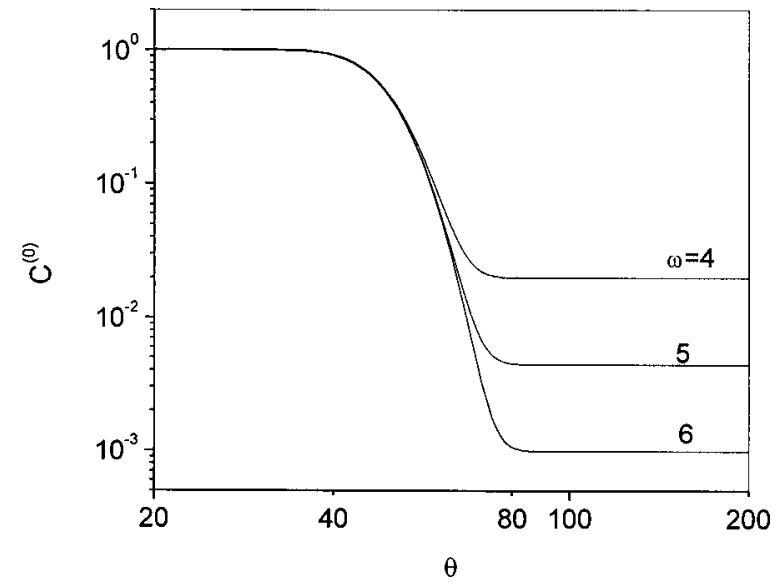

FIG. 8. Effect of $\omega$ on the time variation of the number of clusters. The parameters are $\beta=1, S=2, C_{0}^{\mathrm{avg}}=50$, and $\lambda=\nu=0$.

sociation dominates the rate of denucleation, but association influences the final CSD. The decrease of $C^{(0)}$ with $S$ is consistent with the expectation that as $S$ decreases to its equilibrium value, i.e., unity, the number of unstable clusters will vanish. The effect of $\omega$, the ratio of surface to thermal effects, is similar (Fig. 8); only the final state is influenced by $\omega$. The evolution of the polydispersity is illustrated in Fig. 9 for various values of cluster average size. Regardless of the initial condition, the final polydispersity always approaches unity indicating that almost all the clusters have been converted to the dimer.

The denucleation rate determined above can be compared with the ripening rate calculated from the equations given by Madras and McCoy. ${ }^{7}$ We choose the supersaturation, $S=5$, and $\omega=5$. The critical cluster size, $\xi^{*}$, given by Eq. (2.8), for these values of $S$ and $\omega$, is 30 . The initial average cluster size, $C_{0}^{\text {avg }}$ should be greater than $\xi^{*}$ for ripening and less than $\xi^{*}$ for denucleation. Therefore, the initial condition for ripening and denucleation was chosen to be a delta distribution at $\xi$ equal to 50 and 20, respectively. Figure 10 shows the variation of the cluster numbers with the di-

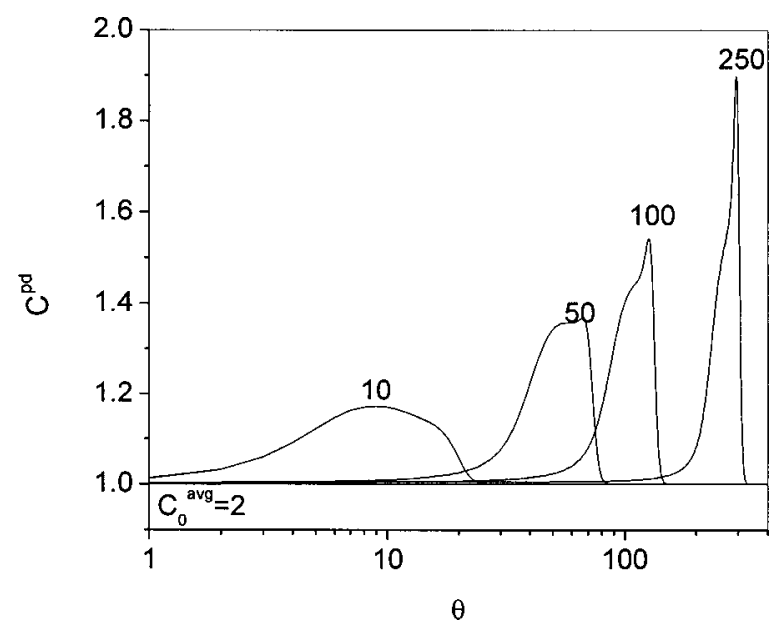

FIG. 9. Effect of the dimensionless initial average cluster size, $C_{0}^{\text {avg }}$, on the time variation of the polydispersity. The parameters are $\beta=1, S=2, C_{0}^{\text {avg }}$ $=50$, and $\lambda=\nu=0$. 


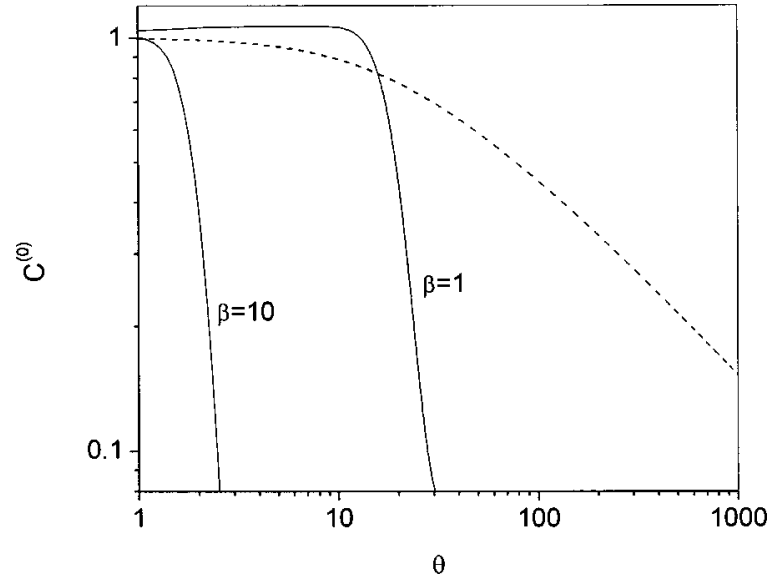

FIG. 10. Variation of the number of clusters with time. The solid line represents the variation due to denucleation with $C_{0}^{\text {avg }}=20$. The dashed line represents the variation due to ripening with $C_{0}^{\mathrm{avg}}=50$. The other parameters are $S=\omega=5$ and $\lambda=\nu=0$.

mensionless time, $\theta$. The figure clearly shows the rate of denucleation is much higher than the ripening rate. This result confirms that denucleation is essentially instantaneous compared to growth and thus supports the hypothesis in our previous papers on ripening. ${ }^{7,23}$

Another striking feature of the study is that the denucleation, though extremely rapid compared to ripening, has an induction period (Fig. 5). Mathematically, this induction occurs because Eq. (3.12) is the governing equation of the system with $C(1, \theta)=S$ and the initial condition, $C(\xi, \theta=0)$ $=\delta\left(\xi=C_{0}^{\text {avg }}\right)$. Equation (3.12) written for the dimer $(\xi=2)$ is dependent on $C(1, \theta)$, which is independent of time when $S$ is assumed to be a constant. The concentration of other clusters rapidly decreases with time and the distribution falls down the stair steps in the energy diagram until reaching $\xi$ $<2$, when it vanishes. Although the distribution is rapidly shifting downward, the number of clusters, $C^{(0)}$, is essentially unchanged until clusters in the distribution reach $\xi$ $=2$. In ripening, the clusters that shrink to the critical size, $\xi^{*}$, immediately begin this descent and quickly disintegrate into monomer.

\section{CONCLUSION}

Founded on concepts of distribution kinetics and governed by population balances, the present theory is simplified by defining scaled quantities. The treatment of denucleation departs from the usual assumptions that stable and unstable clusters in the size distribution are not distinct, and that clusters exist down to zero mass. Ripening behavior can still occur under this approximation because smaller stable clusters are in general more soluble than larger clusters. In prior work we assumed that unstable clusters vanished instantaneously, ${ }^{7}$ that is, very rapidly compared with the rate of growth of stable clusters. Here we have verified that the rate of denucleation is quite rapid relative to stable cluster growth and dissolution. This not only justifies our previous assumption, but also provides insight into this aspect of phase transition dynamics.

The current theory has the advantage that the minimum number of dimensionless parameters and variables is presented explicitly. The mass, $\xi$, and time, $\theta$, dependencies of the CSD, $C(\xi, \theta)$, are governed by the supersaturation, $S$, and the ratio, $\omega$, of cluster surface energy to thermal energy. Rate effects are incorporated in the ratio of dissociation to growth rate parameters, $\beta$, and in the powers on the mass, $\nu$ and $\lambda$, for the respective rate coefficients. We have shown that the power $\nu$ for the dissociation rate coefficient has a predominant effect compared to the power $\lambda$ for the growth coefficient. The initial average cluster size strongly influences the time evolution of number of clusters, with larger clusters having a larger induction time. The supersaturation, $S$, and the ratio of surface to thermal effects, $\omega$, do not influence the denucleation rate but effect the final number of clusters. These computations indicate that dissociation influences the denucleation rate while growth influences the final number of clusters in the CSD.

${ }^{1}$ W. J. Dunning, in Particle Growth in Suspensions, edited by A. L. Smith (Academic, New York, 1973).

${ }^{2}$ J. A. Marqusee and J. Ross, J. Chem. Phys. 79, 373 (1983).

${ }^{3}$ A. W. Adamson and A. P. Gast, Physical Chemistry of Surfaces, 6th ed. (Wiley-Interscience, New York, 1997).

${ }^{4}$ H. Xia and M. Zinke-Allmang, Physica A 261, 176 (1998).

${ }^{5}$ P. Neogi and G. Narsimhan, Chem. Eng. Sci. 56, 4225 (2001).

${ }^{6}$ J. W. Morse and W. H. Casey, Am. J. Sci. 288, 537 (1988).

${ }^{7}$ G. Madras and B. J. McCoy, J. Chem. Phys. 115, 6699 (2001).

${ }^{8}$ J. Frenkel, Kinetic Theory of Liquids (Dover, New York, 1955).

${ }^{9}$ H. Reiss, Science 238, 1368 (1987)

${ }^{10}$ H. Metiu, K. Kitahara, and J. Ross, J. Chem. Phys. 64, 292 (1976).

${ }^{11}$ J. S. Langer, Ann. Phys. (N.Y.) 65, 53 (1971).

${ }^{12}$ N. G. van Kampen, Stochastic Processes in Physics and Chemistry (North-Holland, Amsterdam, 1992).

${ }^{13}$ R. L. Gerlach, J. Chem. Phys. 51, 2186 (1969).

${ }^{14}$ C. H. Yang and H. Liu, J. Chem. Phys. 84, 416 (1986).

${ }^{15}$ B. J. McCoy and G. Madras, Chem. Eng. Sci. 56, 2831 (2001).

${ }^{16}$ R. B. McClurg and R. C. Flagan, J. Colloid Interface Sci. 201, 194 (1998).

${ }^{17} \mathrm{~S}$. Twomey, Atmospheric Aerosols (Elsevier, New York, 1977), Chap. 1.

${ }^{18}$ S. L. Girshick and C.-P. Chiu, J. Chem. Phys. 93, 1273 (1990).

${ }^{19}$ D. W. Oxtoby, J. Phys.: Condens. Matter 4, 7627 (1992).

${ }^{20}$ D. F. Calef and J. M. Deutch, Annu. Rev. Phys. Chem. 34, 493 (1983).

${ }^{21}$ K. Binder, Prog. Inorg. Chem. 15, 4425 (1977).

${ }^{22}$ H. Gratz, J. Mater. Sci. Lett. 18, 1637 (1999).

${ }^{23}$ G. Madras and B. J. McCoy, J. Chem. Phys. (to be published). 